\title{
NUEVO CORONAVIRUS (COVID-19) EN POBLACIÓN GENERAL Y PEDIÁTRICA: UNA REVISIÓN EPIDEMIOLÓGICA. CHILE 2020. NOVEL CORONAVIRUS (COVID-19) IN GENERAL AND PEDIATRIC POPULATION: AN EPIDEMIOLOGICAL REVIEW. CHILE 2020.
}

\section{KIgo. Claudio OImos G. ${ }^{1}$, Dr. Javier Cepeda S. ${ }^{2}$, Dr. Daniel Zenteno A. ${ }^{3}$}

1.- Kinesiologo. PhD (c) en Investigación Biomédica y Salud Pública, MSc Salud Pública, Coordinador Unidad de investigación Clínica INDISA, Docente Escuela de Medicina Universidad Andrés Bello

2.- Pediatra. Hospital Dr. Guillermo Grant Benavente, Concepción. Departamento de Pediatría, Facultad de Medicina, Universidad de Concepción

3.- Pediatra Neumólogo. Hospital Dr. Guillermo Grant Benavente, Concepción. Departamento de Pediatría, Facultad de Medicina, Universidad de Concepción

\section{ABSTRACT}

Since December 2019, the outbreak of a novel coronavirus SARS Cov-2 has been reported in Wuhan, China. Currently, the new coronavirus disease has been declared a worldwide pandemic. Compared to adults, reporting of cases in pediatric patients has been significant smaller. The objective of this article is to provide epidemiological information of COVID-19, especially pediatric. Most of the confirmed cases of children are declared to be a cluster disease. The clinic is oligosymptomatic, less severe and with concentrated risk in children under 1 year of age and with comorbidity. In Chile, pediatric patients represent about $6 \%$ of the total number of infected and overall lethality is significantly lower than adults. The main control measures to reduce effective reproduction are mass testing, social distancing and school closure, without dismissing individual responsibility. The adequate supply of personal protection elements is key to avoid nosocomial infection and the compromise of healthcare providers.

Key Words: COVID-19, epidemiology, children.

\section{RESUMEN}

A partir de diciembre del 2019, se ha reportado el brote de una nueva infección por SARS Cov-2 en Wuhan, China. Actualmente, la enfermedad por el nuevo coronavirus 2019 ha alcanzado el estatus de pandemia. El reporte de casos en pacientes pediátricos ha sido escaso. El objetivo de este artículo es entregar información epidemiológica del COVID-19, especialmente pediátrica. Los niños han presentado enfermedad en clusters, secundaria a contacto con parientes enfermos. La clínica es oligosintomática, menos severa y mayor riesgo concentrado en menores de 1 año y con comorbilidad. En Chile, los pacientes pediátricos representan cerca del 6\% del total y la letalidad global es notablemente más baja que en adultos. Las principales medidas de control para la reducción de la reproducción efectiva son el testeo masivo, distanciamiento social y cierre escolar, sin desestimar la responsabilidad individual. El adecuado abastecimiento de elementos de protección personal es clave para evitar la infección nosocomial y del personal de salud.

Palabras Clave: COVID-19, epidemiología, niños.

\section{INTRODUCCIÓN}

A inicios de diciembre del 2019, fueron reportados casos de neumonía de origen desconocido en Wuhan, provincia de Hubei, China. Secuencias genómicas aisladas de muestras provenientes del tracto respiratorio inferior,

\section{Correspondencia:}

Klgo. Claudio Olmos G.

Escuela de Medicina Universidad Andrés Bello.

Sazié 2320 piso $2^{\circ}$, Santiago, Chile.

Tel: (+56) 226615644.

E-mail: claudio.olmos@unab.cl confirmaron que se trataba de un nuevo tipo de coronavirus circulante(1). En las semanas siguientes, nuevos brotes fueron reportado en Corea del Sur, Irán e Italia. Lo anterior fue seguido rápidamente de múltiples reportes en diversos lugares de Europa, Asia, Norteamérica y diferentes países de Sudamérica(2).

El 7 de enero del 2020, la Organización Mundial de la Salud (OMS) denominó a este patógeno "2019 novel coronavirus (2019-nCoV)". El 30 de enero del 2020, con más de 9700 casos confirmados en China y 106 casos confirmados en otros 19 países, el director general de la OMS declaró el brote como una emergencia de salud pública de 
importancia internacional (ESPII)(3). El 11 de febrero, la OMS, llamó al virus como SARS Cov-2 y la enfermedad causada por este nuevo coronavirus como "coronavirus disease 2019 (COVID-19)". Tras diversas evaluaciones, el 12 de marzo del 2020, la OMS anunció que el COVID-19 había alcanzado el estatus de pandemia(4).

El reporte de casos en población infantil ha sido significativamente menor que en adultos, por lo que hay escasa evidencia focalizada en este grupo etario. En suma, por tratarse de una una cepa particular que no había sido identificada previamente en humanos, existe poca información sobre la historia natural de la enfermedad, epidemiologia e impacto clínico(1,5-7).

El objetivo de esta revisión es reportar las principales características epidemiológicas del COVID-19, dando especial énfasis a población pediátrica.

\section{DESARROLLO DEL TEMA}

Todas las cifras, indicadores y cálculos aquí mencionados, fueron realizados en base a la información oficial existente hasta el 17 de abril, fecha en la que este trabajo fue enviado para su publicación.

Al 16 de Abril, la OMS ha reportado un total de 1.914.916 casos confirmados de COVID-19 y más de 123.000 fallecidos a nivel mundial(8).

Con miles de casos nuevos al día, en todo el globo, los sistemas sanitarios no dieron abasto y el deceso de personas sin poder acceder a intervenciones terapéuticas de alta complejidad, enlutó a naciones de los cinco continentes. En efecto, se estima que durante la primera quincena del mes de abril, cada 5 minutos fallecía una persona infectada con SARS-COV2, en el mundo(8).

Asimismo, los reportes mundiales indican que desde que se declaró oficialmente la pandemia a la fecha de envío del presente trabajo, el reporte de letalidad diaria se ha mantenido relativamente estable, con una pequeña tendencia al alza, con valores que van de un $6,1 \%$ a $7,5 \%$ (Mediana $5,7 \%$; Rango $=3,1 \%-9,1 \%$ ).

En cuanto a la ocurrencia de enfermedad, luego del primer brote que azotó los países del oeste del pacífico, se observó un aumento de casos sostenido en el tiempo, afectando en primera instancia a Europa y luego al continente americano. La figura 1 muestra la incidencia diaria de COVID-19 en el mundo desde que se declaró la enfermedad como una Pandemia.

Actualmente, Ios pacientes con COVID-19 son la principal fuente de infección y aquellos con enfermedad severa son considerados más contagiosos que los casos leves. Los pacientes asintomáticos y aquellos en periodo de incubación, quienes no muestran signos ni síntomas respiratorios y que evidencian eliminación del virus, pueden ser también una fuente potencial de infección(9). A la fecha, existe escasa literatura publicada respecto de la susceptibilidad relacionada con anticuerpos neutralizantes. La evidencia sugiere que cualquiera, independiente de su edad, sexo o raza, que haya tenido contacto estrecho con un individuo infectado, es susceptible al SARS Cov-2(10).

Los pacientes pediátricos son un grupo particular, debido a que su estrecha relación con contactos familiares los hace especialmente susceptibles a infección cruzada. De acuerdo con la información epidemiológica existente, la mayoría de los casos en niños con COVID-19, demuestran evidencia de transmisión a través de encuentros familiares, comportándose como una enfermedad en clusters o "infección de segunda generación", sin reportes claros que evidencien a niños siendo la fuente de infección de adultos $(4,11)$.

Dong y cols., realizaron un análisis epidemiológico de los pacientes pediátricos con COVID-19 en China. Se registraron un total de 2135 casos, de los cuales 1407 $(65,9 \%)$ fueron sospechosos y $728(34,1 \%)$ confirmados por laboratorio. La edad media de los pacientes fue 7 años (Rango Intercuartílico: 2 años - 13 años) sin diferencias significativas en el número de varones y mujeres. En consideración a la gravedad, 94 (4,4\%) fueron asintomáticos, $1088(51,0 \%)$ leves y $826(38,7 \%)$ moderados, siendo en suma un $94,1 \%$ del total. Entre los pacientes con enfermedad severa y aquellos críticamente enfermos, los menores de un año representaron la mayor proporción de casos $(10,6 \%)$ con relación al total de pacientes confirmados de este grupo etario, seguido por aquellos entre 1 y 5 años $(7,3)$ y 6 a 10 años $(4,2 \%)$. Se reportó 1 fallecimiento de un adolescente de 14 años. El número medio de días desde el inicio de la enfermedad al diagnóstico fue de 2 días (rango: 0 - 42 días), siendo la mayoría diagnosticados en la primera semana de síntomas. La distribución espacial de los casos pediátricos siguió una clara tendencia hacia una rápida expansión desde Hubei a las provincias y ciudades cercanas a través del tiempo por sobre áreas más alejadas(1).

Por su parte, el reporte semanal de morbilidad y mortalidad del equipo de respuesta al COVID-19 del Center for Disease Control and Prevention (CDC), publicado el 10 de abril del 2020, informó un total de $2.572(1,7 \%)$ casos de menores de 18 años dentro de un universo de 149.082 confirmados. El primer paciente pediátrico con COVID-19 fue reportado por el CDC el 2 de marzo del 2020 y desde el 5 de marzo, nuevos casos se han presentado en forma diaria. La información recopilada fue incompleta en diversas variables estudiadas. De un total de 2.490 pacientes con sexo conocido, $1.408(57 \%)$ fueron de sexo masculino. La edad media fue de 11 años (rango: 0 - 17 años). Entre aquellos con información completa, un $73 \%$ de los pacientes pediátricos presentaron fiebre, tos o dificultad respiratoria comparado con un $93 \%$ de adultos entre 18 y 64 años durante el mismo periodo. Las mialgias, odinofagia, cefalea y diarrea fueron también menos frecuentes en la población pediátrica. Entre aquellos en quienes se conocía su situación de exposición, un 91\% estuvo 
asociado a exposición a un paciente positivo para SARS Cov2 en su domicilio o comunidad y un $9 \%$ se relacionó con un viaje reciente. Un total de $147(5,7 \%)$ pacientes pediátricos requirieron hospitalización ( $20 \%$ de todos aquellos en quienes se conocía su situación de hospitalización), un porcentaje menor a los adultos hospitalizados entre los 18 y 64 años (10\%). A su vez, en 15 casos de menores de 18 años se requirió ingreso a $\mathrm{UCI}(0,58-2 \%)$, cifra menor a la observada en población adulta (1,4-4,5\%). Los menores de 1 año son el mayor porcentaje de pacientes hospitalizados en la población pediátrica $(15-62 \%)$. Del total de pacientes con información de comorbilidad, un 23\% presentaron alguna condición subyacente y en aquellos que requirieron hospitalización, un $77 \%$ presentaba comorbilidades conocidas. Las comorbilidades más frecuentes fueron: enfermedad pulmonar crónica - incluyendo asma - $(50 \%$ del total), seguido de enfermedad cardiovascular e inmunosupresión. A la fecha, solo se han reportado 3 pacientes pediátricos fallecidos en todo el mundo. Estos datos apoyan hallazgos previos de que el COVID-19 en niños se presenta de manera oligosintomática en comparación al adulto, y que la mayoría de los casos pediátricos no son severos, sin embargo, igualmente presentan un riesgo de requerir hospitalización(12,13).

En Chile, a la fecha han ocurrido 116 muertes atribuibles al COVID-19, exhibiendo una letalidad nacional del 1,25; notablemente más baja que la cifra mundial.

De los 92 fallecidos, el 43,9\% pertenece a la región Metropolitana y un 19,4\% a la región de la Araucanía (Tabla 1).

En cuanto a la ocurrencia de enfermedad en Chile, el primer caso confirmado en Chile se notificó el 2 de marzo de 2020. Se trataba de casos importados, identificándose tres grupos familiares. Un primer grupo familiar correspondió a cuatro personas que viajaron a Italia y Reino Unido. Entre ellos estaban dos hermanos de 14 y 17 años que dieron positivo el 5 de marzo, transformándose en los primeros casos en población menor de edad en el país. Posteriormente un segundo grupo familiar, compuesto por dos personas de la región del Maule que viajaron al sudeste asiático y Europa, generaron dos casos asociados en Chile. Uno de ellos una niña de 2 años de edad (8 de Marzo).

Al 14 de abril del 2020, se había confirmado un total de 8273 casos de COVID-19 y de ellos, 271 correspondían a casos en menores de 15 años(14). En las figuras 2 y 3 se observan la incidencia acumulada de COVID-19, por día, para población pediátrica y población general.

La mediada de edad del total de casos confirmados en Chile es de 40 años, concentrándose el 71,6\% de los casos, en personas entre 25 y 59 años. Las mayores tasas de incidencia acumulada, según casos confirmados por laboratorio en la población general, se encuentran en la región de Magallanes (248,9 x 100.000 hab.), Ñuble (123,9 x 100.000 hab.), La Araucanía (81,4 x 100.000 hab.), a las que le siguen las re giones Metropolitana, Arica y Parinacota y los Lagos con poco más de 40 casos por cada cien mil habitantes; todas por sobre la tasa nacional (tabla 2)(14).

El número de casos y tasa de incidencia acumulada de casos confirmados de COVID-19 en la población pediátrica se describen en la tabla 3.

El reporte epidemiológico nacional no hace referencia a las manifestaciones clínicas, hospitalizaciones ni ingreso a camas críticas relacionados con la población pediátrica, sin embargo, la Sociedad Chilena de Pediatría, por medio de su rama de intensivo Pediárico, informa que a nivel país, al día 15 de abril se hallaban 7 pacientes pediátricos hospitalizados con diagnóstico de COVID-19; ninguno de ellos en Unidad de Tratamientos Intensivos.

Otro aspecto epidemiológico que ha cobrado especial interés en esta pandemia, es la velocidad de transmisión y la posibilidad de controlar el brote. Ahí es donde recobran importancia los modelamientos matemáticos que permiten entender la dinámica de la transmisión. Para ello, la epidemiología recurre a elementos técnicos como el $\mathrm{R}(0)$ o ritmo básico de reproducción, que es el número de infecciones nuevas por unidad de tiempo multiplicado por el tiempo que una persona infectada puede contagiar, y describe el número de infecciones nuevas que se originaron del individuo infectado inicialmente $(15,16)$.

Asimismo, también se describe otro valor conocido como $R$ efectivo, $R(e)$, el cual corresponde al $R(0)$ multiplicado por la proporción de individuos susceptibles(16).

Si el número de reproducción efectiva, $R(e)$, es mayor que 1,0, cabe prever que la enfermedad se siga propagando; la reproducción efectiva refleja el hecho de que, a medida que la proporción de individuos susceptibles disminuye, la transmisión de la enfermedad se vuelve más lenta. Basándose en esta perspectiva matemática sencilla, los epidemiólogos suelen considerar que el número reproductivo básico es uno de los parámetros decisivos para determinar si una epidemia es susceptible de control $(17,18)$.

El objetivo de toda respuesta de salud pública durante una pandemia, consiste en aminorar o detener la propagación del virus mediante estrategias de mitigación que: 1) disminuyan el $R(0)$ mediante la disminución en la probabilidad de transmisión (por ejemplo, cerrando las escuelas) 0 bien 2) disminuyan el $\mathrm{R}(\mathrm{e})$, reduciendo el número de individuos susceptibles (por ejemplo, mediante la vacunación si es que existe esa posibilidad)(16).

El día 17 de Marzo de 2020, el Doctor Mauricio Canals, académico del programa de Salud Ambiental de la Escuela de Salud Pública de la Universidad de Chile, realizó una presentación a los presidentes y representantes de partidos políticos de todo el espectro, con la finalidad de explicar el ingreso de Chile a fase 4 a través de las modelaciones de la pandemia de COVID-19 que vive el mundo. En dicha ocasión, se hizo notar que países como China, Corea y Singapur lograron "quebrar" la curva epidémica, con $\mathrm{R}(0)$ cercanos 0 menores que 1; Japón esta mitigando el crecimiento de la epidemia con un $\mathrm{R}(0)$ aproximado de 1.5. Otros países como Italia y España no lo lograron y su epidemia crece con R0 mayores que el resto del mundo, colapsando sus sistemas 
de salud . Si bien, aún no se cuenta con cifras definitivas, se estima que, tanto los brotes observados en China como en la mayor parte de los países de Europa, tuvieron valores de $\mathrm{R}(0)$ superiores a 2, lo que implica que las medidas mundiales han hecho muy poco efecto sobre la capacidad de dispersión del virus $(15,19,20)$.

\section{DISCUSIÓN Y CONCLUSIONES}

Con todo lo expuesto, se puede concluir que la Pandemia por SARS Cov-2, nos plantea un escenario epidemiológico que debe ser combatido con medidas esenciales. La eficacia de las intervenciones no farmacológicas implementadas en todo el globo, varía según la oportunidad y precocidad con que se hayan ejecutado.

El testeo masivo y las intervenciones focalizadas en los individuos infectantes, hasta ahora han formado la pieza central de los esfuerzos de control en algunos lugares, como Singapur y Hong Kong(21). Muchos otros países están adoptando medidas de "distanciamiento social" 0 "distanciamiento físico", cerrando escuelas y lugares de trabajo y limitando el tamaño de las reuniones. El objetivo de estas estrategias es reducir la intensidad máxima de la epidemia ("aplanar la curva")(21), reducir el riesgo de abrumar los sistemas de salud y ganar tiempo para desarrollar tratamientos y vacunas(22).

Para que el distanciamiento social haya revertido la epidemia en China, el número de reproducción efectiva debe haber disminuido al menos en un 50-60\%, suponiendo un valor de referencia R0 entre 2 y 2.5 (21). Mediante medidas de control intensivo, Shenzhen pudo reducir el número de reproducción efectiva en un $85 \%$ estimado(23). Es posible que las medidas de distanciamiento social deban durar meses para controlar efectivamente la transmisión y mitigar la posibilidad de resurgimiento(24).

Con respecto a las medidas de control adoptadas en la población pediátrica, se realizó una revisión sistemática para identificar que se conoce respecto de la efectividad del cierre escolar y otras medidas de distanciamiento social escolar durante brotes de coronavirus. Se incluyeron un total de 9 estudios. Ninguno de estos, a excepción de los estudios de modelamiento, fue diseñado para evaluar específicamente medidas de distanciamiento escolar, por lo que la calidad de la evidencia es baja. Datos provenientes de brotes de SARS en China, Hong Kong y Singapur, sugieren que la transmisión escolar no juega un rol importante en el brote y medidas como el cierre escolar o control de temperatura, no contribuyen al control de la infección. No obstante, un estudio de modelamiento en población británica específicamente diseñado para evaluar medidas de distanciamiento social escolar, estimó que un escenario de cierre escolar completo y de un $25 \%$ de las universidades, era capaz de reducir entre un $2-4 \%$ el total de muertes. El aislamiento individual fue la medida única más importante y la mejor, la combinación de ambas estrategias $(21,25)$.

También parece justo decir que el comportamiento individual juega un rol crucial para controlar la propagación del SARS Cov-2. El autoaislamiento temprano, búsqueda de asistencia médica remota a menos que los síntomas sean severos y el distanciamiento social depende en gran parte de la ciudadanía(21). Aquello parece uno de los factores claves que explicarían la distribución desigual de la enfermedad a lo largo del territorio nacional. En consecuencia, los gobiernos deben vigilar posibles focos de transmisión y entregar las condiciones para facilitar el aislamiento efectivo de casos.

Otra problemática a considerar en esta discusión es la transmisión intrahospitalaria del SARS Cov-2, que se ha reportado aún mayor que lo observado en anteriores brotes de coronavirus como fueron el Síndrome respiratorio agudo grave (SARS) de 2002 y el síndrome respiratorio de Oriente Medio (MERS), afectando particularmente al personal médico. Un reciente estudio retrospectivo indicó que un total de 1716 trabajadores de la salud estaban infectados, lo que representa el $3.84 \%$ del total de casos. Las infecciones nosocomiales del personal de salud agobiaron en gran medida el sistema de salud e impidieron que los pacientes con infección temprana recibieran asistencia médica inmediata, lo que resultó en una alta tasa de letalidad en Wuhan $(12,26)$. La evidencia sugiere que la falta de equipo de protección personal (EPP) y/o equipamientos inadecuados sería una de las principales causa de muerte por SARS Cov-2 entre los trabajadores de la $\operatorname{salud}(27)$.

Mirando hacia el futuro, algunos estudios ya señalan que habrán brotes estacionales recurrentes de SARS-CoV-2 durante el invierno, por lo que, de no existir nuevas medidas terapéuticas eficientes como una vacuna, podrá ser necesario aplicar medidas de distanciamiento social prolongado 0 intermitente en 2021, para evitar que se excedan las capacidades de atención crítica(22).

No cabe duda que la detección de casos y la reducción de contacto han demostrado ser altamente eficaz si se inician precozmente, impidiendo una mayor propagación. Para ello, contar con un sistema de vigilancia eficiente, con cobertura nacional y gestión centralizada, es clave. Esto también implica necesariamente, una comunicación efectiva tanto a nivel de entidades sanitarias, como para con la ciudadanía.

Así, las estrategias deben prepararse, adoptarse y ajustarse de manera que se pueda minimizar los impactos sanitarios, sociales y económicos en las regiones afectadas alrededor del Mundo. Asimismo, urge que las autoridades locales, se aseguren de entregar las medidas de protección adecuadas al personal de salud, para evitar que éstos enfermen, situación que podría incrementar el progreso de la pandemia.

Finalmente, sería importante recalcar la urgencia con la que se necesitan estudios serológicos longitudinales para determinar el alcance y la duración de la inmunidad al SARSCoV-2. Incluso en el caso de una eliminación aparente, la vigilancia del SARS-CoV-2 debe mantenerse ya que un resurgimiento del contagio podría ser posible hasta 2024(22).

Los autores declaran no tener conflictos de interés 
Figura 1. Incidencia diaria de COVID-19 en el mundo.

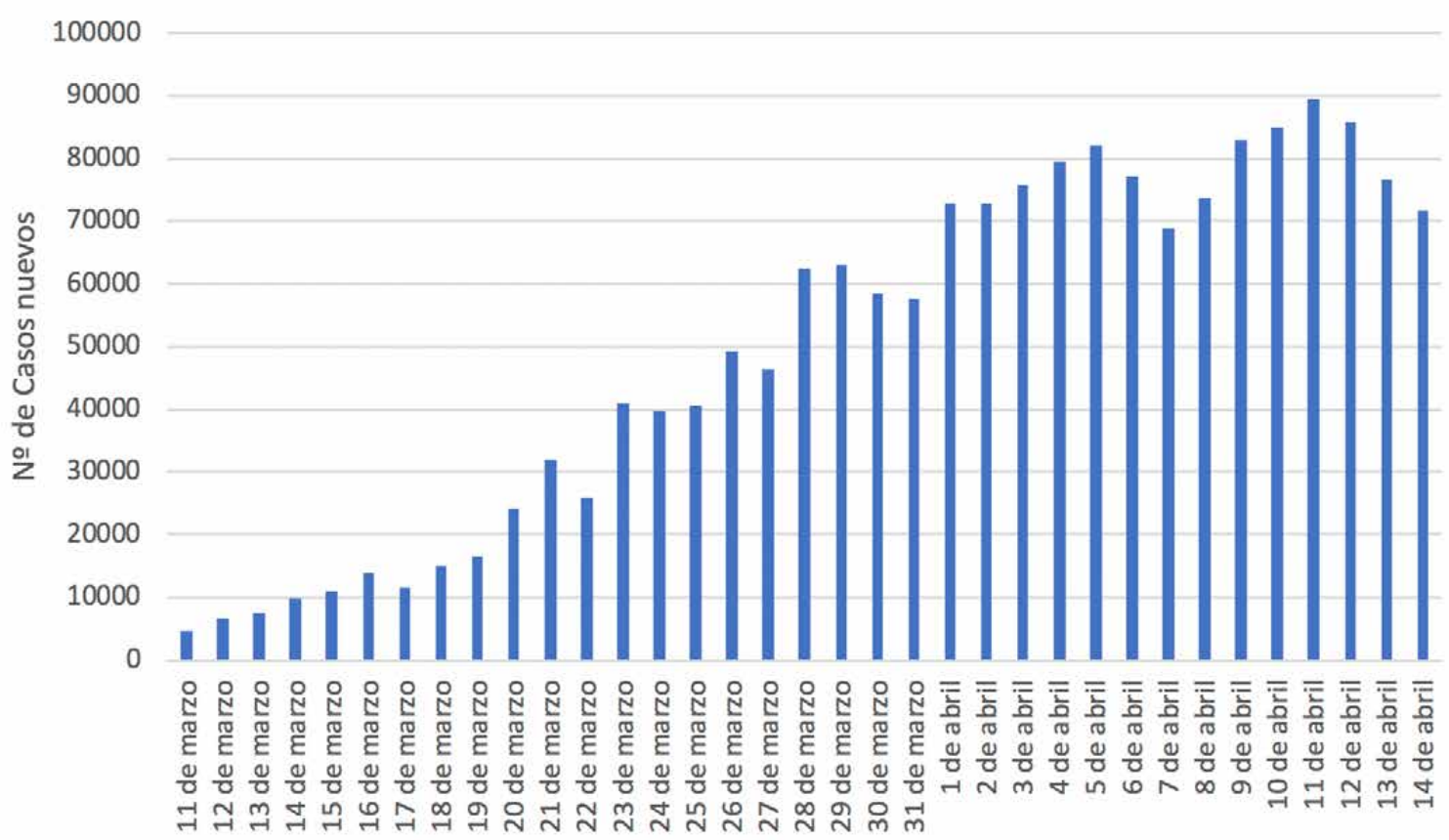

Fuente: Creación propia de los autores. Basado en los Informes de situación COVID-19. Organización mundial de la salud.

Figura 2. Incidencia Acumulada de COVID-19, por día, en población general y población pediátrica, Chile 2020.

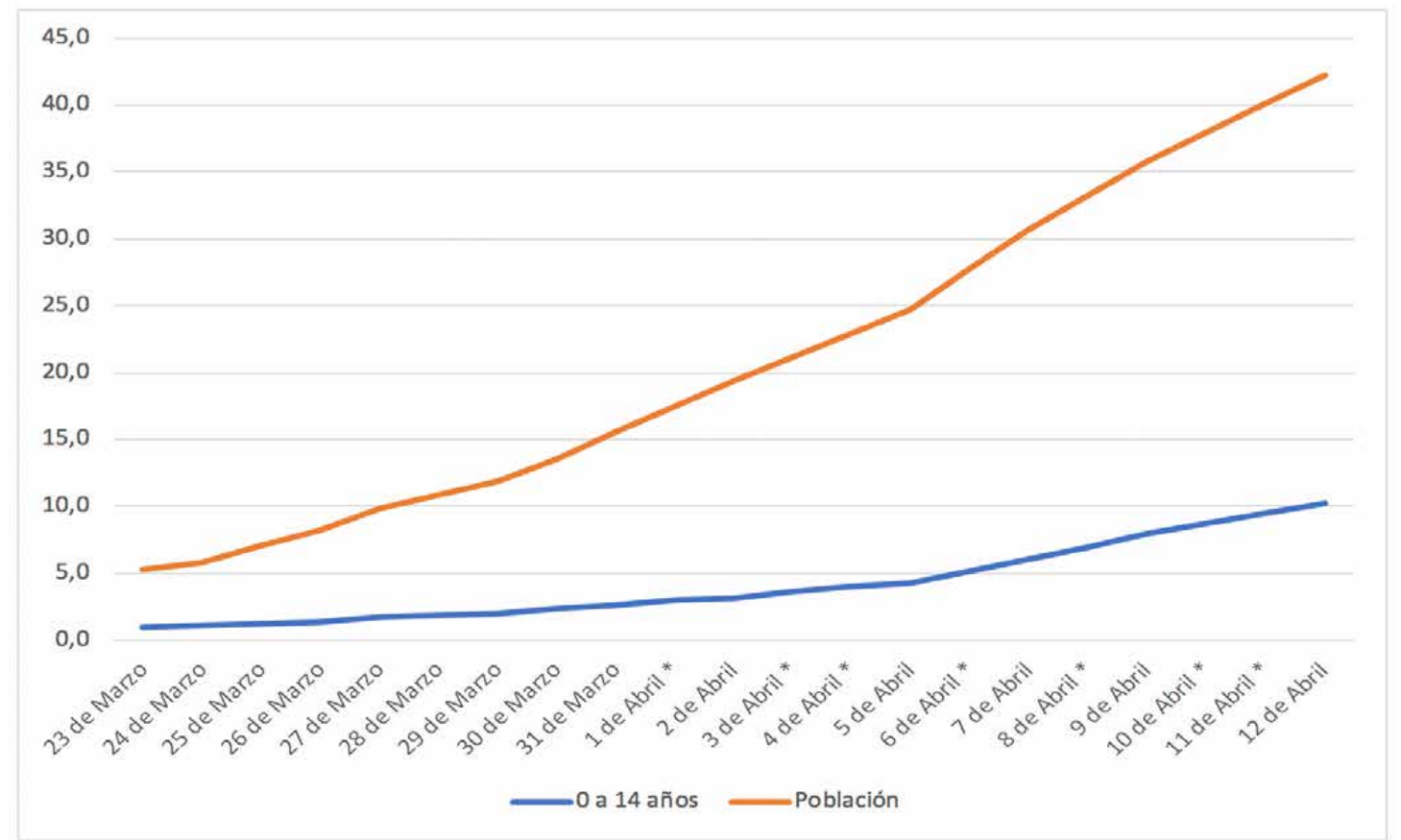

Fuente: Creación propia de los autores. Basado en los Informes de situación COVID-19. Departamento de Epidemiología. Ministerio de Salud de Chile. La población utilizada para el cálculo de incidencia, fue la reportada por el Censo 2017.

(*) Las fechas con este símbolo, no cuentan con informe oficial publicado, por lo que su valor corresponde a la media entre los dos puntos disponibles. 


\section{Figura 3.}

Incidencia Acumulada

de COVID-19, por día,

en población pediátrica,

desglosada por grupo

etario Chile 2020.

Fuente: Creación propia de los autores. Basado en los Informes de situación COVID-19. Departamento de Epidemiología. Ministerio de Salud de Chile.

La población utilizada para el cálculo de incidencia, fue la reportada por el Censo 2017.

$\left.{ }^{*}\right)$ Las fechas con este símbolo, no cuentan con informe oficial publicado, por lo que su valor corresponde a la media entre los dos puntos disponibles.

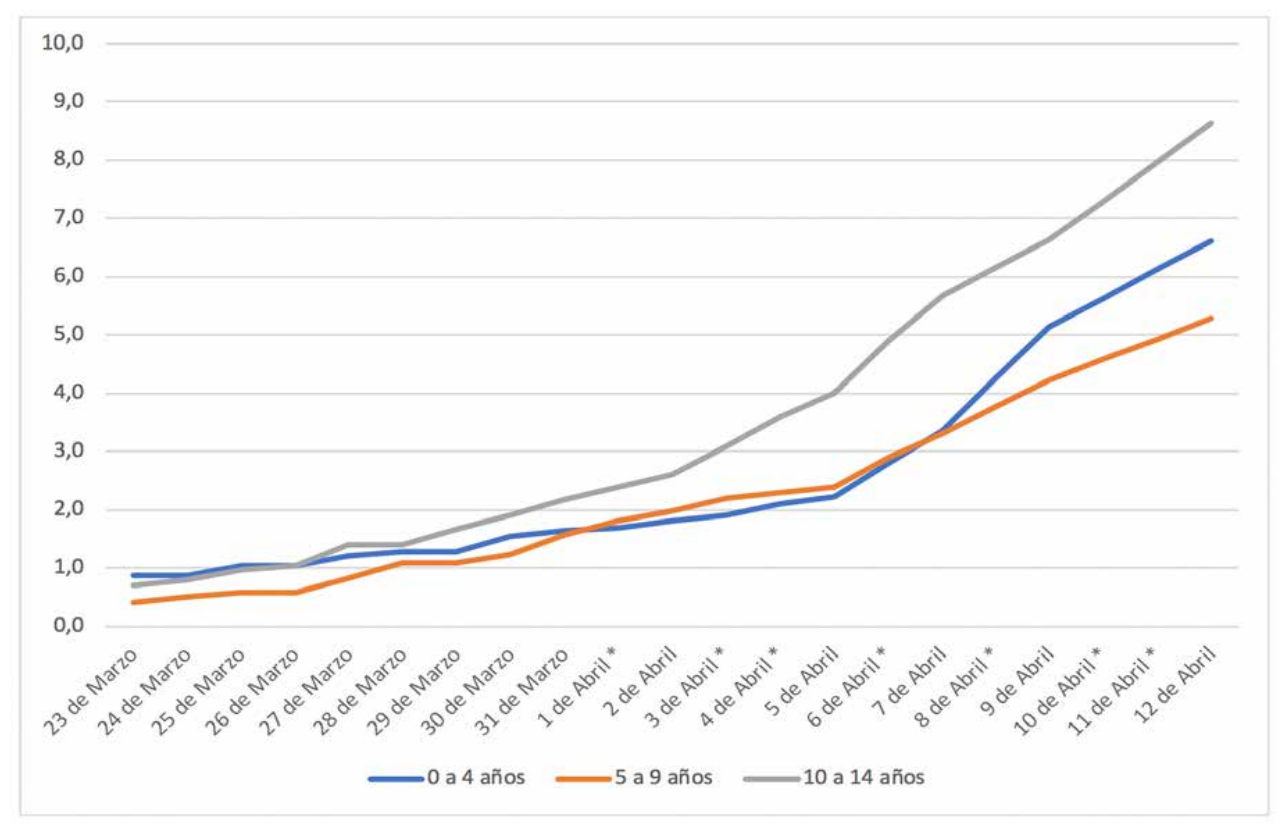

Tabla 1. Casos confirmados de COVID-19 y número de fallecidos por dicha causa, según región. 14 de Abril de 2020, Chile.
Fuente: Basado en los Informes de situación COVID-19. Departamento de Epidemiología. Ministerio de Salud de Chile.

\begin{tabular}{lllll} 
& \multicolumn{2}{c}{ Casos totales } & \multicolumn{2}{l}{ Fallecidos } \\
Región & $\mathbf{n}$ & $\mathbf{\%}$ & $\mathbf{n}$ & $\mathbf{\%}$ \\
\hline Arica y Parinacota & 120 & $1,52 \%$ & 1 & $1,1 \%$ \\
Tarapacá & 62 & $0,78 \%$ & 0 & $0,0 \%$ \\
Antofagasta & 155 & $1,96 \%$ & 1 & $1,1 \%$ \\
Atacama & 13 & $0,16 \%$ & 0 & $0,0 \%$ \\
Coquimbo & 66 & $0,83 \%$ & 0 & $0,0 \%$ \\
Valparaíso & 285 & $3,60 \%$ & 2 & $2,2 \%$ \\
Metropolitana & 4.086 & $51,61 \%$ & 40 & $43,5 \%$ \\
O’Higgins & 53 & $0,67 \%$ & 0 & $0,0 \%$ \\
Maule & 142 & $1,79 \%$ & 4 & $4,3 \%$ \\
Nuble & 622 & $7,86 \%$ & 8 & $8,7 \%$ \\
Biobío & 528 & $6,67 \%$ & 2 & $2,2 \%$ \\
Araucanía & 816 & $10,31 \%$ & 20 & $21,7 \%$ \\
Los Ríos & 148 & $1,87 \%$ & 3 & $3,3 \%$ \\
Los Lagos & 385 & $4,86 \%$ & 5 & $5,4 \%$ \\
Aysén & 7 & $0,09 \%$ & 0 & $0,0 \%$ \\
Magallanes & 429 & $5,42 \%$ & 6 & $6,5 \%$ \\
& & & & \\
\hline Total & $\mathbf{7 . 9 1 7}$ & $\mathbf{1 0 0 \%}$ & $\mathbf{9 2}$ & $100 \%$ \\
& & & &
\end{tabular}


Tabla 2. Número de casos y tasa de incidencia de COVID-19 según región de confirmación diagnóstica. Chile, acumulado al 14 de abril de 2020.

\begin{tabular}{|lcc|}
\hline Región & $\begin{array}{c}\text { Número de } \\
\text { Casos } \\
\text { Confirmados }\end{array}$ & $\begin{array}{c}\text { Tasa incidencia } \\
\text { acumulada }\end{array}$ \\
\hline Arica y Parinacota & 124 & 49,2 \\
Tarapacá & 62 & 16,2 \\
Antofagasta & 176 & 25,4 \\
Atacama & 13 & 4,1 \\
Coquimbo & 66 & 7,9 \\
Valparaíso & 299 & 15,3 \\
Metropolitana & 4.334 & 53,3 \\
O'Higgins & 54 & 5,4 \\
Maule & 152 & 13,4 \\
Nuble & 634 & 123,9 \\
Biobío & 542 & 32,6 \\
Araucanía & 826 & 81,4 \\
Los Ríos & 150 & 37,0 \\
Los Lagos & 390 & 43,7 \\
Aisén & 7 & 6,5 \\
Magallanes & 444 & 248,9 \\
Total país & $\mathbf{8 . 2 7 3}$ & $\mathbf{4 2 , 5}$ \\
\hline
\end{tabular}

Tabla 3. Número de casos y tasa de incidencia acumulada de casos de COVID-19 en menores de 20 años, notificados según grupo de edad y sexo. Chile, acumulado al 14 de abril.

\begin{tabular}{|c|c|c|c|c|c|c|}
\hline \multirow[b]{2}{*}{$\begin{array}{l}\text { Grupo } \\
\text { etario }\end{array}$} & \multicolumn{2}{|c|}{ Hombre } & \multicolumn{2}{|l|}{ Mujer } & \multicolumn{2}{|l|}{ Total } \\
\hline & $\begin{array}{c}\text { Casos (\% del } \\
\text { total nacional) }\end{array}$ & Tasa* & $\begin{array}{c}\text { Casos }(\% \text { del } \\
\text { total nacional) }\end{array}$ & Tasa* & $\begin{array}{c}\text { Casos (\% del } \\
\text { total nacional) }\end{array}$ & Tasa* \\
\hline $\begin{array}{l}00-04 \\
\text { años }\end{array}$ & $46(1,1)$ & 7,6 & $46(1,1)$ & 7,9 & $92(1)$ & 6,5 \\
\hline $\begin{array}{l}05-09 \\
\text { años }\end{array}$ & $32(0,8)$ & 4,9 & $40(1)$ & 6,4 & $72(0,9)$ & 5,6 \\
\hline $\begin{array}{l}10-14 \\
\text { años }\end{array}$ & $57(1,4)$ & 8,9 & $50(1,2)$ & 8,0 & $107(1,3)$ & 8,5 \\
\hline $\begin{array}{l}15-19 \\
\text { años }\end{array}$ & $86(2,1)$ & 13,4 & $121(2,9)$ & 19,5 & $207(2,5)$ & 16,4 \\
\hline
\end{tabular}




\section{REFERENCIAS}

1. Dong $Y$, Mo X, Hu Y, Qi X, Jiang F, Jiang Z. Epidemiology of COVID-19 Among Children in China. 2020;145(6).

2. Hasan A, Mehmood N, Fergie J. Coronavirus Disease (COVID-19) and Pediatric Patients: A Review of Epidemiology, Symptomatology, Laboratory and Imaging Results to Guide the Development of a Management Algorithm. Cureus. 2020;12(3).

3. Internacional RS, Comit E, General E, Houssin PD. Declaración sobre la segunda reunión del Comité de Emergencias del Reglamento Sanitario Internacional ( 2005 ) acerca del brote del nuevo. 2020;(2005):1-7.

4. Jiatong $\mathrm{S}$, lanqin $\mathrm{L}$, Wenjun L. COVID-19 epidemic: disease characteristics in children. J Med Virol. 2020;0-2.

5. Ciotti M, Angeletti S, Minieri M, Giovannetti M, Benvenuto D, Pascarella S, et al. COVID-19 Outbreak: An Overview. Chemotherapy [lnternet]. 2020;1-9. Available from: http://www. ncbi.nlm.nih.gov/pubmed/32259829

6. Hong H, Wang Y, Chung HT, Chen CJ. Clinical characteristics of novel coronavirus disease 2019 (COVID-19) in newborns, infants and children. Pediatr Neonatol [Internet]. 2020;61(2):131-2. Available from: https://doi.org/10.1016/j.pedneo.2020.03.001

7. WHO (World Health Organization). Similarities and differences - COVID-19 and influenza. 2020;(March):17-9. Available from: https://www.who.int/news-room/q-a-detail/q-a-similaritiesand-differences-covid-19-and-influenza

8. World Health Organization (WHO). Coronavirus disease 2019 (COVID-19) Situation Report - 86. World Heal Organ [Internet]. 2020;(April):2633. Available from: https://www.who.int/ emergencies/diseases/novel-coronavirus-2019

9. Jin Y, Yang H, Ji W, Wu W, Chen S, Zhang W, et al. Virology, epidemiology, pathogenesis, and control of covid-19. Viruses. 2020;12(4):1-17.

10. Park M, Cook AR, Lim JT, Sun Y, Dickens BL. A Systematic Review of COVID-19 Epidemiology Based on Current Evidence. J Clin Med. 2020;9(4):967.

11. Huang X, Wei F, Hu L, Wen L, Chen K. IRANIAN Epidemiology and Clinical Characteristics of COVID-19. Acad Med Sci IR Iran [Internet]. 2020;23(4):268-71. Available from: https://doi. org/10.34172/aim.2020.09

12. Team CC-19 R. Coronavirus Disease 2019 in Children United States, Februar 12-April 2, 2020. Morb Mortal Wkly Rep [Internet]. 2020;69(56):1-8. Available from: https:// www.cdc.gov/mmwr/volumes/69/wr/mm6914e4.htm?s cid=mm6914e4_w\#suggestedcitation

13. Korioth T, Writer S. CDC : Children with COVID-19 less likely to be hospitalized, show symptoms than adults. 2020;2019.

14. Departamento De Epidemiología M de S. Informe Epidemiológico: Enfermedad por SARS-CoV-2 (COVID-19). Chile 17-04-2020.

15. Riou J, Althaus CL. Pattern of early human-to-human transmission of Wuhan 2019 novel coronavirus (2019nCoV), December 2019 to January 2020. Eurosurveillance. 2020;25(4):1-5.

16. Ridenhour B, Kowalik JM, Shay DK. Public Health Applications. Am J Public Heal [Internet]. 2014;104(2):32-41. Available from: http://www.ajph.org

17. Fisman DN, Moghadas S, Day T, Bauch C, Driedger SM, Brauer F, et al. Modelling an influenza pandemic: A guide for the perplexed - Pandemic Influenza Outbreak Research Modelling Team (PanInfORM). Cmaj. 2009;181(3-4):171-3.

18. Pellis L, Ferguson NM, Fraser C. Threshold parameters for a model of epidemic spread among households and workplaces. J R Soc Interface. 2009;6(40):979-87.

19. Sanche S, Lin YT, Xu C, Romero-Severson E, Hengartner N, Ke R. High Contagiousness and Rapid Spread of Severe Acute Respiratory Syndrome Coronavirus 2. Emerg Infect Dis [nternet]. 2020;26(7):1-19. Available from: http://www.ncbi.nlm.nih.gov/ pubmed/32255761

20. Kucharski AJ, Russell TW, Diamond C, Liu Y, Edmunds J, Funk $S$, et al. Early dynamics of transmission and control of COVID-19: a mathematical modelling study. Lancet Infect Dis. 2020;3099(20):1-7.

21. Anderson RM, Heesterbeek H, Klinkenberg D, Hollingsworth TD. How will country-based mitigation measures influence the course of the COVID-19 epidemic? Lancet. 2020;395(10228):931-4.

22. Kissler SM, Tedijanto C, Goldstein E, Yonatan G, Lipsitch M. Projecting the transmission dynamics of SARS-CoV-2 through the postpandemic period. Science (80- ). 2020;1-18.

23. Qifang B, Yongsheng W, Shujiang M, Chenfei Y, Xuan Z, Zhen Z, et al. Epidemiology and Transmission of COVID-19 in Shenzhen China: Analysis of 391 cases and 1,286 of their close contacts. medRxiv. 2020;

24. Lai S, Ruktanonchai NW, Zhou L, Prosper O, Luo W, Floyd JR, et al. outbreak in China. 2020;1-29.

25. Viner RM, Russell SJ, Croker H, Packer J, Ward J, Stansfield $\mathrm{C}$, et al. School closure and management practices during coronavirus outbreaks including COVID-19: a rapid systematic review. Lancet Child Adolesc Heal [Internet]. 2020;0(0):1-8. Available from: https://linkinghub.elsevier.com/retrieve/pii/ S235246422030095X

26. Wang D, Hu B, Hu C, Zhu F, Liu X, Zhang J, et al. Clinical Characteristics of 138 Hospitalized Patients with 2019 Novel Coronavirus-Infected Pneumonia in Wuhan, China. JAMA - J Am Med Assoc. 2020;323(11):1061-9.

27. Edsel I, Xu A, Salimi A, Torum N. Physician Deaths from Corona Virus Disease (COVID-19). medRxiv. 2020; 